\title{
Super-Resolution of Digital Images Using CNN with Leaky ReLU
}

\author{
Jebaveerasingh Jebadurai, Immanuel Johnraja Jebadurai, Getzi \\ Jeba Leelipushpam Paulraj, Nancy Emymal Samuel
}

\begin{abstract}
Image super-resolution (SR) has been used in many real world applications as a preprocessing phase. The improvement in image resolution increases the performance of image analysis process. The $S R$ of digital images is achieved by taking the low resolution images as inputs. In this article, a novel deeplearning based super-resolution approach is proposed. The proposed approach uses Convolutional Neural Network (CNN) with leaky rectified linear unit (ReLU) for learning and generalization. The experiments with test images taken from USC-SIPI dataset indicate that the proposed approach increases the quality of the images in terms of the quantitative metric peak signal to noise ratio.
\end{abstract}

Keywords--- Super-Resolution, Deep Learning, Convolutional Neural Network, Leaky ReLU.

\section{INTRODUCTION}

Image super-resolution is the process of increasing the spatial resolution of digital images. Since, the high resolution (HR) images possess more useful information than that of low resolution ones, HR images are preferred over the LR images in many practical applications [1]. The resolution of the digital images can be improved in two hardware modalities. The other one is by the application of any image processing techniques. Making changes to the hardware restrictsthe area of application. Whereas, the image processing techniques can be applied to the digital images independent of the image capturing devices. In addition to that, the resolution of low resolution images can be improved effectively by these image processing techniques. Image super-resolution is one such technique that improves the spatial resolution of low resolution images.

Over the years several SR techniques have been proposed. This paper proposes a deeplearning based image SR technique for the low resolution digital images. The proposed approach uses CNN with leaky ReLU as the activation function. The results of the experiments have been evaluated with the quantitative measure PSNR.

The remainder of the article is organized as follows. The study about the literature for the various SR approaches are given in Section 2. The proposed SR-CLR approach is explained in section 3. The experimental setup and the results are discussed in Section 4. The conclusion and the

Manuscript received September 16, 2019.

Jebaveerasingh Jebadurai, Computer Science and Engineering, mail: jebaveerasingh.j@gmail.com)

Immanuel Johnraja Jebadurai, Computer Science and Engineering, Karunya Institute of Technology and Sciences, Coimbatore, T.N, India.(email: immanueljohnraja@gmail.com)

Getzi Jeba Leelipushpam Paulraj, Computer Science and Engineering, Karunya Institute of Technology and Sciences, Coimbatore, T.N, India. (e-mail: getz23@gmail.com)

Nancy Emymal Samuel, System Administrator, Sam Salt Works, Tuticorin, T.N, India. (e-mail: s.nancyemymal@gmail.com) different ways. The first is by making changes to the Karunya Institute of Technology and Sciences, Coimbatore, T.N, India.(e-

future scope are given in Section 5 followed by the list of references.

\section{RELATED WORKS}

Over the years, several approaches have been proposed for increasing the spatial resolution of digital images. The most frequently used techniques are the image interpolation techniques like nearest neighbor, bilinear and bicubic interpolations. Most of the image editing applications use bicubic interpolation to increase the resolution due to its time complexity. However, the quality of the resultant image will be entirely dependent on the input image. The sparse coding based approaches used by the researchers produced less number of non-zero entries during the construction of high resolution images. An additional refinement process is required in these approaches $[2,3]$.

The super-resolution approaches are found to be applicable invariably in all the areas of applications where the digital images are used. These SR approaches can be grouped into two large classifications namely single-frame SR and multi-frame SR. The single frame approaches use a single source LR images. The multi-frame approaches, on the other hand, require several images of the same scene. The SR approaches are further classified in to reconstruction based approaches, which focus on removing the aliasing artifacts, and learning based approaches which form relationships among the low resolution and high resolution pairs of images. The reconstruction based approaches perform well when the scaling factor, also known as the magnification factor, is below 2. The learning based approaches have used several methods for learning the relationships among the patches. There has been feature pyramid based learning, learning with belief networks, projection based learning and learning with the help of neural networks $(\mathrm{NN})[4,5]$. The application of these learning based approaches have helped in generating better high resolution images than the reconstruction based approaches.

\section{PROPOSED SR-CLR APPROACH}

The aim of the proposed approach is to generate higher resolution image from the input low resolution image. The input image is taken as the ground truth image for performance evaluation. The learning is achieved by the convolutional neural network.

The flow of the proposed SR-CLR is depicted in fig.1.

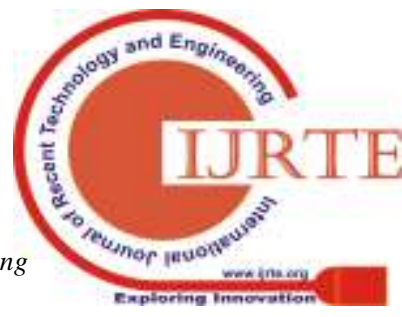




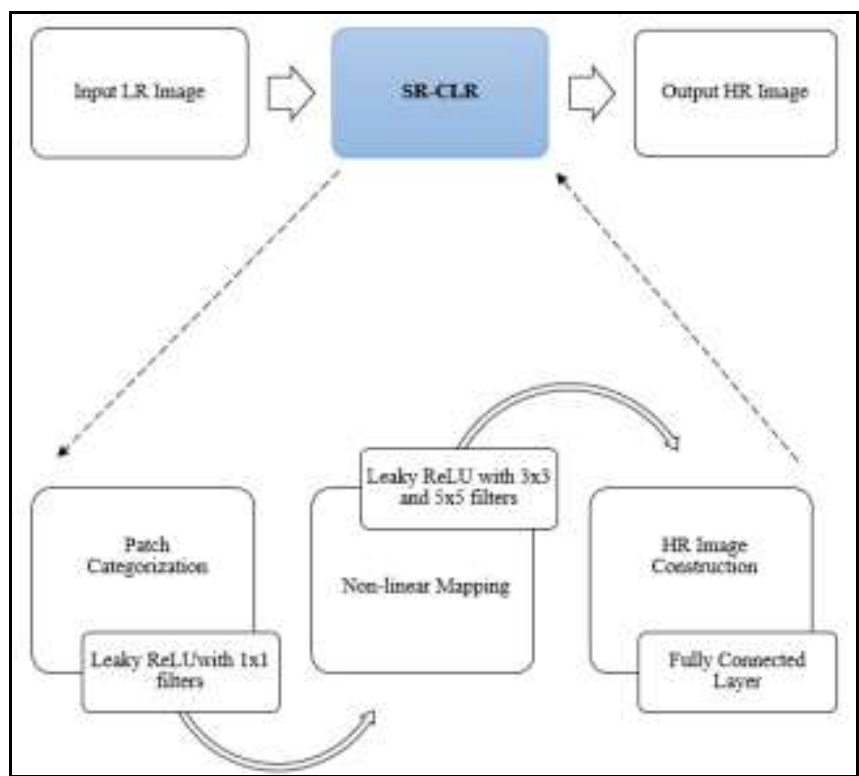

Fig. 1: Flow of SR-CLR Approach

The convolutional neural network in the proposed approach has three layers, namely categorization of patches, non-linear mapping and construction of HR image. The first two layers use, Leaky ReLU, expressed in (1), is used as the activation function in the CNN of the SR-CLR approach.

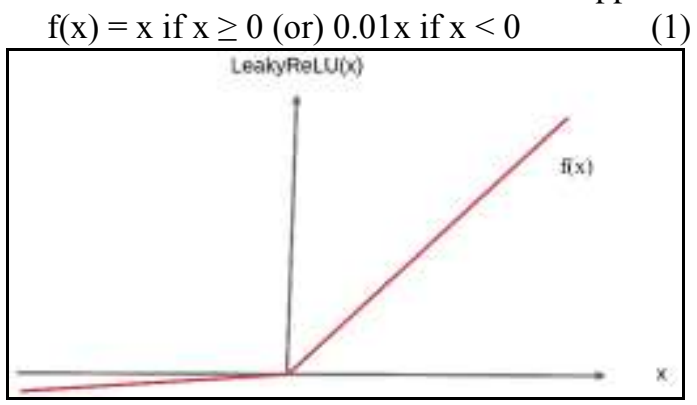

Fig. 2: Leaky ReLU

The major advantages of using Leaky ReLU over ReLU are that, it overcomes the limitation of dying ReLU and supports higher learning rates [6].

The filter size of $1 \times 1$ is used in the categorization of the patches. The process of patch categorization is expressed in (2). Here, $\mathrm{f} 1$ is the filter of size $1 \mathrm{x} 1$ and $\mathrm{b}$ is the bias.

$$
1(\mathrm{x})=\max (0.01 \mathrm{x}, \mathrm{f} 1 * \mathrm{x}+\mathrm{b})
$$

The overlapping image patches are formed as the result of the non-linear mapping. Here, the proposed SR-CLR approach uses the filter sizes of $3 \times 3$ or $5 \times 5$. The expression given in (3) gives the operation of non-linear mapping.

$$
11(\mathrm{x})=\max (0.01 \mathrm{x}, \mathrm{f} 2 * 1(\mathrm{x})+\mathrm{bb})
$$

Here, $\mathrm{f} 2$ is the filter of size $3 \times 3$ and $5 \times 5$ and bb is the bias.

The final layer constructs of the expected high resolution image. It is achieved by averaging all the overlapping patches together. This averaging operation is expressed in (4).

$$
h(x)=f 3 * 11(x)+b b b
$$

Here, $\mathrm{h}(\mathrm{x})$ represents the HR image, f3is the filter and bbb is the bias. The SR-CLR approach is an in-scale superresolution technique that produces the $\mathrm{HR}$ image with the size same as that of the input LR image.

\section{EXPERIMENTAL SETUP \& RESULTS}

The proposed approach was implemented and tested with various input images taken from the USC-SIPI datasets [7] and the outputs were tabulated. Similarly, the other SR approaches such as bicubic interpolation, self-learning based SR (SLSVR) [8], sigmoid kernel SVR based SR (SKSVR) [9], deep convolutional neural network based SR (DCNN) [4] and Hybrid CNN-SVM [10] approaches were also implemented and the outputs were taken. The learned outcomes with ImageNet dataset were used for the learning [11]. The performance of the approaches is compared based on the metric PSNR.

The test images are depicted in Fig.3. The experiments were carried out for five times for each test images. The average of PSNR values achieved from the experiments are listed in table 2 .

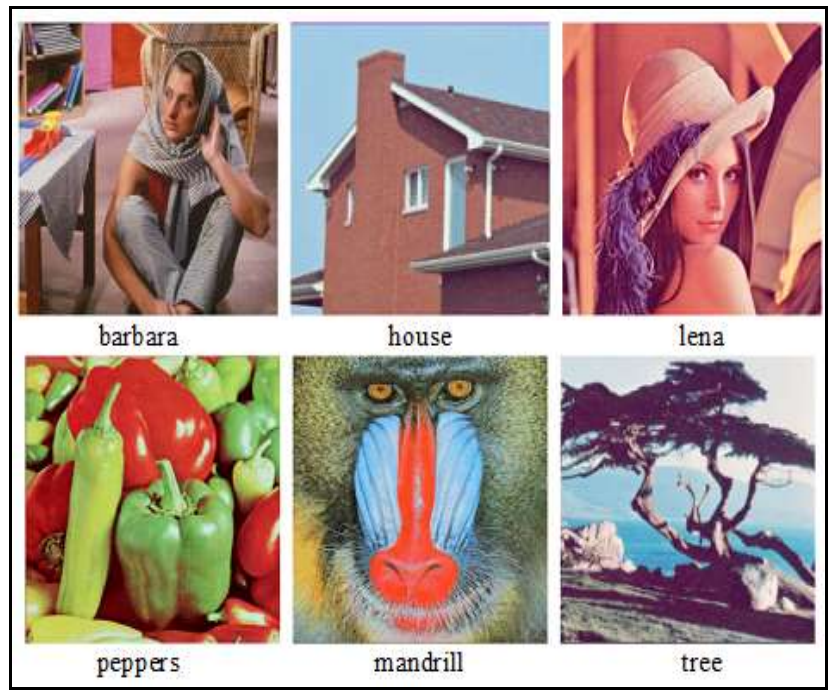

Fig. 3: Test Images from the USC-SIPI Dataset

\begin{tabular}{|c|c|c|c|c|c|c|}
\hline \multirow{2}{*}{$\begin{array}{l}\text { SR } \\
\text { Metho } \\
\text { ds }\end{array}$} & \multicolumn{6}{|c|}{ Test Images } \\
\hline & $\begin{array}{l}\text { barbar } \\
a\end{array}$ & $\begin{array}{l}\text { hous } \\
e\end{array}$ & lena & $\begin{array}{l}\text { peppe } \\
\text { rs }\end{array}$ & $\begin{array}{l}\text { mandri } \\
l l\end{array}$ & tree \\
\hline Bicubic & 29.72 & $\begin{array}{l}29.3 \\
3\end{array}$ & $\begin{array}{l}31.8 \\
3\end{array}$ & 29.83 & 29.82 & $\begin{array}{l}25.6 \\
1\end{array}$ \\
\hline $\begin{array}{l}\text { SLSVR } \\
{[8]}\end{array}$ & 30.42 & $\begin{array}{l}30.0 \\
7 \\
\end{array}$ & $\begin{array}{l}32.5 \\
8 \\
\end{array}$ & 29.96 & 30.06 & $\begin{array}{l}26.4 \\
2 \\
\end{array}$ \\
\hline $\begin{array}{l}\text { SKSV } \\
\text { R [9] }\end{array}$ & 30.44 & $\begin{array}{l}30.0 \\
9 \\
\end{array}$ & $\begin{array}{l}32.6 \\
0 \\
\end{array}$ & 30.38 & 30.14 & $\begin{array}{l}26.4 \\
5 \\
\end{array}$ \\
\hline $\begin{array}{l}\text { DCNN } \\
{[4]}\end{array}$ & 30.68 & $\begin{array}{l}30.3 \\
8 \\
\end{array}$ & $\begin{array}{l}33.7 \\
2 \\
\end{array}$ & 31.46 & 30.32 & $\begin{array}{l}27.6 \\
7 \\
\end{array}$ \\
\hline $\begin{array}{l}\text { Hybrid } \\
\text { CNN- } \\
\text { SVM } \\
{[10]}\end{array}$ & 30.74 & $\begin{array}{l}30.3 \\
6\end{array}$ & $\begin{array}{l}33.8 \\
5\end{array}$ & 31.48 & 30.38 & $\begin{array}{l}27.5 \\
7\end{array}$ \\
\hline $\begin{array}{l}\text { SR- } \\
\text { CLR }\end{array}$ & 30.76 & $\begin{array}{l}30.3 \\
6\end{array}$ & $\begin{array}{l}33.9 \\
2\end{array}$ & 31.54 & 30.42 & $\begin{array}{l}27.7 \\
1\end{array}$ \\
\hline
\end{tabular}
Table 1: PSNR Values (in Decibels) obtained from the Experiments

It can be identified from the values of Table I that the proposed SR-CLR approach performs better than the state of the art image super-resolution approaches. The high resolution results obtained from the SR approaches are depicted in fig. 4 .

It is evident from fig. 4 that, the proposed SR-CLR approach produces better high resolution images in terms of quantitative measure as well as visual perception. 


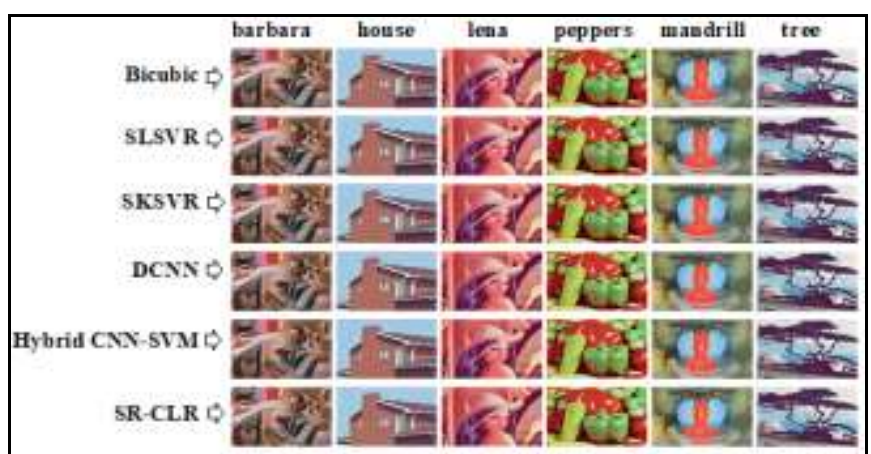

Fig. 4: High Resolution Images Obtained from the Experiments

\section{CONCLUSION AND FUTURE SCOPE}

The SR-CLR approach for the enhancement of compressed images has been proposed. The proposed approach used deep learning for producing the learned outcomes. The application of leaky ReLU activation function helped the SR-CLR approach to produce better results. The performance evaluation based on the quantitative metric PSNR indicated that the proposed approach outperforms the state of the art SR approaches. The future works will be based on the optimization of the proposed SR approach in terms of learning time and the development of SR approaches for IoT based applications.

\section{ACKNOWLEDGMENT}

The authors thankfully acknowledge the computational facilities provided by Karunya Institute of Technology and Sciences for doing research in this subject.

\section{REFERENCES}

1. Hayit Greenspan, Super-Resolution in Medical Imaging, The Computer Journal, Vol. 52, no. 1, pp 43-63. January 2009.

2. K. I. Kim and Y. Kwon, "Single-image superresolution using sparse regression and natural image prior," IEEE Trans. Pattern Anal. Mach. Intell., vol. 32, no. 6, pp. 1127-1133, Jun. 2010.

3. J. Yang, J. Wright, T. S. Huang, and Y. Ma, "Image super-resolution via sparse representation," IEEE Trans. Image Process., vol. 19, no. 11, pp. 2861-2873, Nov. 2010.

4. Dong, C., Loy, C. C., He, K., \& Tang, X. (2016). Image super-resolution using deep convolutional networks. IEEE transactions on pattern analysis and machine intelligence, 38(2), 295-307.

5. K. He, X. Zhang, S. Ren, and J. Sun, "Spatial pyramid pooling in deep convolutional networks for visual recognition," in Proc. Eur. Conf. Comput. Vis., 2014, pp. 346-361.

6. S.-H. Wang, P. Phillips, Y. Sui, B. Liu, M. Yang, and H. Cheng, "Classification of Alzheimer's Disease Based on Eight-Layer Convolutional Neural Network with Leaky Rectified Linear Unit and Max Pooling," Journal of Medical Systems, vol. 42, no. 5, 2018.

7. SIPI Image Database [Online]. Available: http://sipi.usc.edu/database/. [Accessed: 13-May2019]

8. Min-Chun Yang Yu-Chiang Frank Wang, A SelfLearning Approach to Single Image Super-Resolution, IEEE Trans. Multimedia, vol. 15, no. 3, pp. 498-508, Apr. 2013.
9. J. Jebadurai and J. Peter, "SK-SVR: Sigmoid kernel support vector regression based in-scale single image super-resolution", Pattern Recognition Letters, vol. 94, pp. 144-153, 2017.

10. J. Jebadurai and J. D. Peter, "Deep CS: Deep Convolutional Neural Network and SVM Based Single Image Super-Resolution," Lecture Notes in Computer Science, vol. 11076, pp. 3-13, 2018.

11. A. Krizhevsky, I. Sutskever, and G. Hinton, "ImageNet classification with deep convolutional neural networks," in Proc. Adv. Neural Inf. Process. Syst., 2012, pp. 1097-1105. 